\title{
Framework of Dynamic Resource Allocation System for Virtual Machine in Virtualization System
}

\author{
D. Jannarm and T. Kaewkiriya
}

\begin{abstract}
The purpose of this research is to design of concept dynamic resource allocation system for virtual machine in virtualization system. The process of this design is assigning the basic resource to virtual machine as well as designing the modules for monitoring and detecting the resource usage in virtual machine for each time period in order to allocate the resource suitably by simulate three situations of using the resource. The first situation is the used resource is equal to initial resource without adding additional resource. The second situation is the used resource is more than initial resource but it can add more resource. Finally, the third situation is the used resource is less than initial resource and the leftover resource can be regained. According to this proposed allocation resource, it is very helpful for allocating the resource in Virtualization system effectively because the system will detect the resource for each time period in real time. Also, the system can allocate and recover the unused resource depend on real usage.
\end{abstract}

Index Terms-Resource management, virtual machine, virtualization system.

\section{INTRODUCTION}

Nowadays, Virtualization system [1] is being used by many organizations especially in data center which can be vastly useful for data management because Virtualization system is to combine multiple servers to exist in one device which is called "Sever Consolidation [2]." Also, Virtualization technology enable IT infrastructure to be more flexible by allowing Virtualize Machine in Virtualization system to be able to share resource from the same hardware. However, using the resources on each Virtual machine has to depend on the time period of usage and all applications on Virtual machine. It makes the usage of resource in physical hardware can be inconstant all the time. Thus, a capability of allocation of resource on Virtual machine should be considered about appropriate resource with high performance for applications which run on Virtual Machine.

That means the resources have to be determined based on usage. If the resources are defined more than necessity, it will waste the resource. On the other hand, if the resource is determined less than usage, the performance of system in providing applications on Virtual machine will be ineffective which comes from insufficient resource.

According to the resource adjustment and "Live migration [3]" (the process of running virtual machine from one physical server to another) it brings the combination of theory to control a variety of usability in order to enhance the

Manuscript received November 20, 2014; revised May 5, 2015.

The authors are with the Faculty of Information Technology, Thai-Nichi Institute of Technology, and Information \& Communication Center Bangkok, Thailand (e-mail: dolwichit@tni.ac.th, thongchai@tni.ac.th). performance of resource allocation to be suitable for the use of Virtual machine, applications and the resource allocation between Virtual machine and physical server in Virtualization system such as Vmware, Xen which contain the same controller for sharing resource. For example, in the process of sharing CPU, there are Credit scheduler [4] for management and Balloon driver [5] for RAM usage management. However, in the present, the resource assignments on Virtual machine are defined constantly which can highly occur errors. Thus, the preventing of this case is determining amount of resource in Virtual machine more than real usage in order to support each workloads in each virtual machine. Also, this amount of resources can be calculated from prediction of service such as database server, web server and others services which are located on each Virtual machine.

After analysis the dynamic resource allocation system for virtual machine has been identified to have the modules as follows; 1) Resource sensor module 2) Resource detection module 3) Resource regulator module 4) Resource Management module 5) Resource provider/reclaimer.

This paper mainly consists of six main sections: first: Introduction Second: Review related research. Third: Related theory forth: Conceptual framework fifth: Simulation experiments sixth: Conclusion and Guideline for future research.

\section{RELATED WORK}

Previously, Gang Ning et al. [6] had studied about resource allocation on Xen hypervisor and presented its methods which divided into 3 levels (by priority) as follows; 1. Core application level 2. Service application level 3.Common application level as the result of method, the core application level was the fastest provider. Moreover, Minaroli et al. [7] used Fuzzy to predict the requirement of resource on Virtual machine by inventing Fuzzy controller for checking on the performance and making a discussion of resource management. Nigmandjanovich et al. [8] studied through an experiment. As an experiment, they set up agent on Virtual machine for monitoring resource usage and identified Policy-base to each service in order to prioritize the access of resource. Minarolli et al. [9] presented Distribution as the method of resource management through artificial neural network. Also, they used artificial neural network as a principle of learning. Ying Song et al. [10] demonstrated Distribution sharing resource between Physical machine in Data center and presented Algorithm in Distribution which distinguished into two types; local and global. Wenyu Zhou et al. [11] suggested resource allocation for Virtual Machine cluster by using load balance. Load balance was used to 
distribute resource on each Virtual machine cluster at the same domain. Chao-Tung Yang et al. [12] utilized the principle of the cluster to share resource by producing a cluster at a physical host to balance workload.

However, according to previous researches, it shows that they analyzed the resource allocation base on real resource usage on Virtual machine and applications in order to specify Rule and Priority. Also, they used control theory to help them predict and create a model for producing a controller.

This research has presented a model of resource allocation with dynamic work as follows. The first section) is for monitoring resource usage. The second section) is used for detecting the use of resource. The third section) is to check on the demand of resource. The forth section) is resource allocation. The fifth section) is to notify the resource usage to Virtual machine besides the system can be able to allocate resource and restore some resource as needed for each time period.

\section{BACKGROUND}

Virtualization technology is to imitate a virtual version of resource in computer such as CPU, Ram and storage. With Virtualization, a single computer can allow the system hardware to run multiple different operating systems at the same time. This makes Virtual machine to work as a completely independent computer which installs on hardware. Currently, there are a lot of virtualization software [13] for business and also provides as open source without expense. Virtualization can be classified into three categories which are following.

\section{A. Full Virtualization}

As shown in Fig. 1, full virtualization [14] or native virtualization is a process that make an entire computer system into a software construct. This software construct performs like the original hardware in every way. As performed by software construct, it is no need to modify hardware which executes on the Virtual machine. Full virtualization uses a special software called a Hypervisor to work through Virtual machine monitoring (VMM) as Binary transaction to Virtual machine.

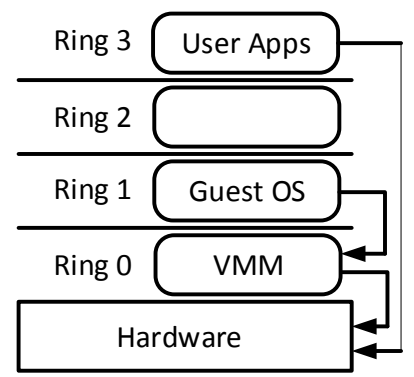

Fig. 1. Infrastructure of full virtualization.

\section{B. Paravirtualization}

Paravirtualization [15] is the collaboration between Virtual machine and Hypervisor to provide more effective performance. In paravirtualization system, hypervisor can modify non-virtualize hardware inside Virtual machine or kernel by an intermediary between Virtual machine and
Hypervisor to make the entire system can communicate and work together like a cohesive unit as in Fig. 2.

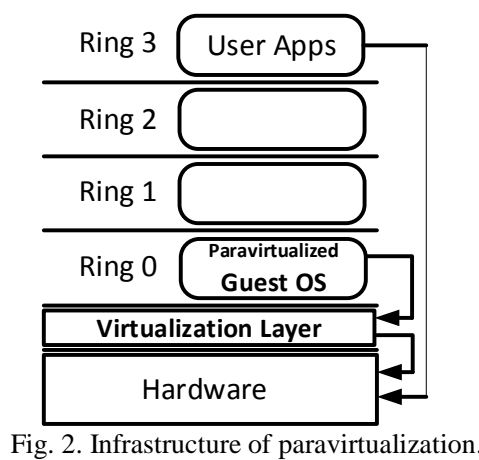

\section{Hardware Assisted Virtualization}

As shown in Fig. 3, hardware assisted virtualization [16] is the development of hardware technology to support and simplify Virtualization techniques. For instance, Intel Virtualization- Technology (VT-x) and AMD's AMD-V are targeted to maximize the effectiveness of Virtual machine monitoring (VMM) in term of Binary translation of Full virtualization and Paravirtualization.

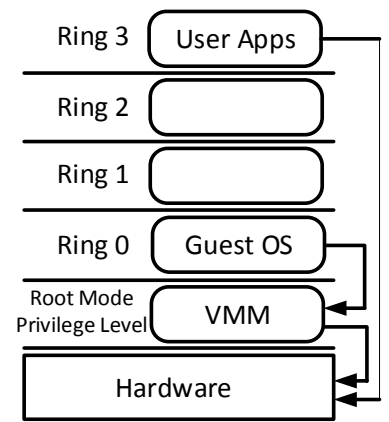

Fig. 3. Infrastructure of hardware assisted virtualization.

\section{CONCEPTUAL FRAMEWORK}

This research introduces the concept of resource allocation system in Virtualization system as follows: In general, each virtual machine is configured to use the default resource. After that, it is a process for monitoring resource of Virtual machine at each time period in order to determine whether the system needs more resources or use fewer resources. This process helps to allocate and restore resource from Virtual machine depends on demand. Also, the conceptual framework of resource allocation is shown in Fig. 4.

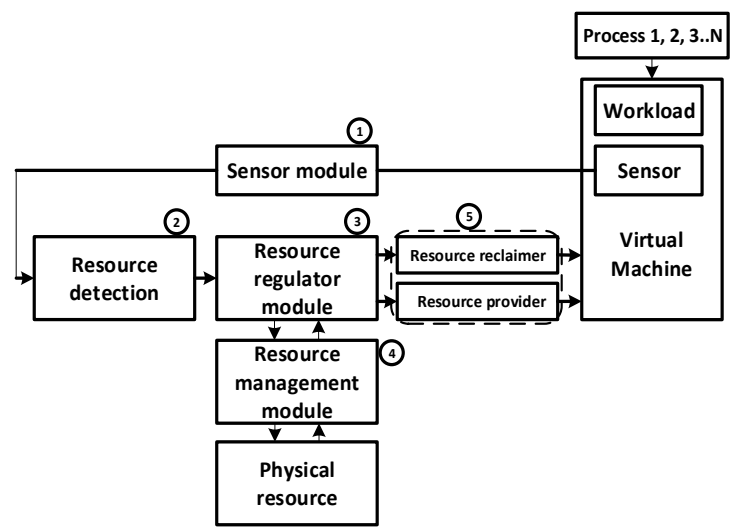

Fig. 4. Conceptual framework. 
The main function module of resource allocation:

Resource sensor module: This module used for monitoring resource of Virtual machine in real time.

Resource detection module: This module is used for detecting resource usage and specify the source that which virtual machine it comes from.

Resource regulator module: This module is to control and set the rules for resource allocation and restoration.

Resource management module: This module is about resource management and the hypervisor interaction for resource requirement and restoration.

Resource provide/reclaim: This module is to inform the resource allocation and restoration value to virtual machine.

According to above design concept, workload which comes out from monitoring is placed on CPU and RAM and this is used for allocation of virtual machine resource on the step of resource detection on RAM and CPU. In this step, resource detection identifiers virtual machine information such as specify which machine that it comes from and uses this information as a process condition to define criteria of allocation and recovery resource for defining condition, researcher uses Fuzzy logic as a process condition Fig. 5.

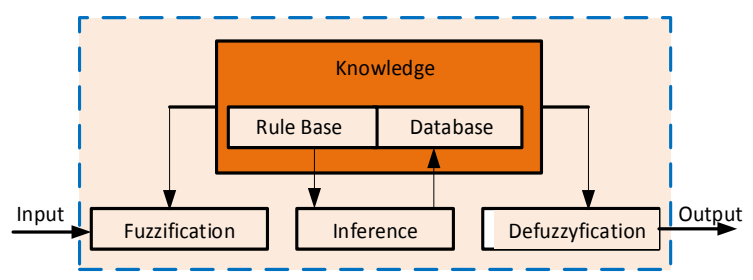

Fig. 5. Fuzzy logic process condition.

In order to determine of requirement by using Fuzzy logic, it can assist in monitoring the needs of the individual Virtual machine resources allocation which it should add more or decrease resource based on appropriate usage by set input as Time interval and information resource of virtual machine as following Fig. 6.

According to Fig. 6(A) is input data which is the resource usage of each time interval; T1, T2, T3 ... Tn. Also, this input data comes from Sensor module and Resource Detection module for indicate the number of Virtual machine and type of resources and then forward these information to Resource regulator module to use Fuzzy logic

Fig. 6(B) is to verify Virtual machine whether there is a need to use the resources. In this session, an expert can define Rule base as following:

IF CPU Equal Initial.Res(CPU), RAM Equal Initial.Res(RAM) THEN NOT Allocate

IF CPU More Than Initial.Res(CPU), RAM Equal Initial.Res(RAM) THEN Allocate CPU

IF CPU Less Than Initial.Res(CPU), RAM Equal Initial.Res(RAM) THEN Reclaim CPU

IF CPU Equal Initial.Res(CPU), RAM More Than Initial.Res(RAM) THEN Allocate RAM

IF CPU Equal Initial.Res(CPU), RAM Less Than Initial.Res(RAM) THEN Reclaim RAM

IF CPU More Than Initial.Res(CPU), RAM More Than Initial.Res(RAM) THEN Allocate CPU, RAM

IF CPU More Than Initial.Res(CPU), RAM Less Than
Initial.Res(RAM) THEN Allocate CPU, Reclaim RAM

IF CPU Less Than Initial.Res(CPU), RAM More Than Initial.Res(RAM) THEN Reclaim CPU, Allocate RAM IF CPU Less Than Initial.Res(CPU), RAM Less Than Initial.Res(RAM) THEN Reclaim CPU, Reclaim RAM

To forward to the Resource management module for determine the percentage of usage and inform Virtual machine monitoring (VMM) to request the resource usage on Hardware section.

\begin{tabular}{cc|c} 
& CPU $_{\text {usage(\%) }}$ & RAM usage(MB) \\
\cline { 2 - 3 } $\mathrm{T} 1$ & 5 & 800 \\
\cline { 2 - 3 } $\mathrm{T} 2$ & 6 & 600 \\
\cline { 2 - 3 } $\mathrm{T} 3$ & 15 & 1200 \\
\hline $\mathrm{T} 4$ & 10 & 800 \\
\hline $\mathrm{T} 5$ & 12 & 1000 \\
\hline
\end{tabular}

(A) Resource usage interval time

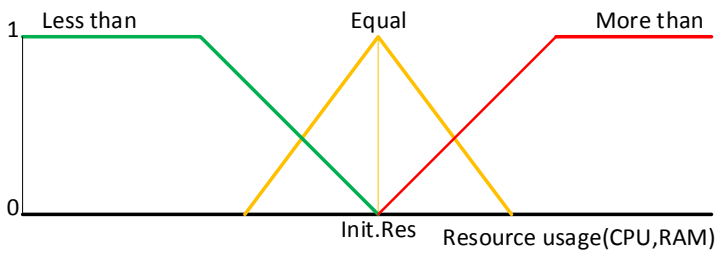

(B) Resource usage membership function

Fig. 6. Resource usage interval time and resource usage membership function.

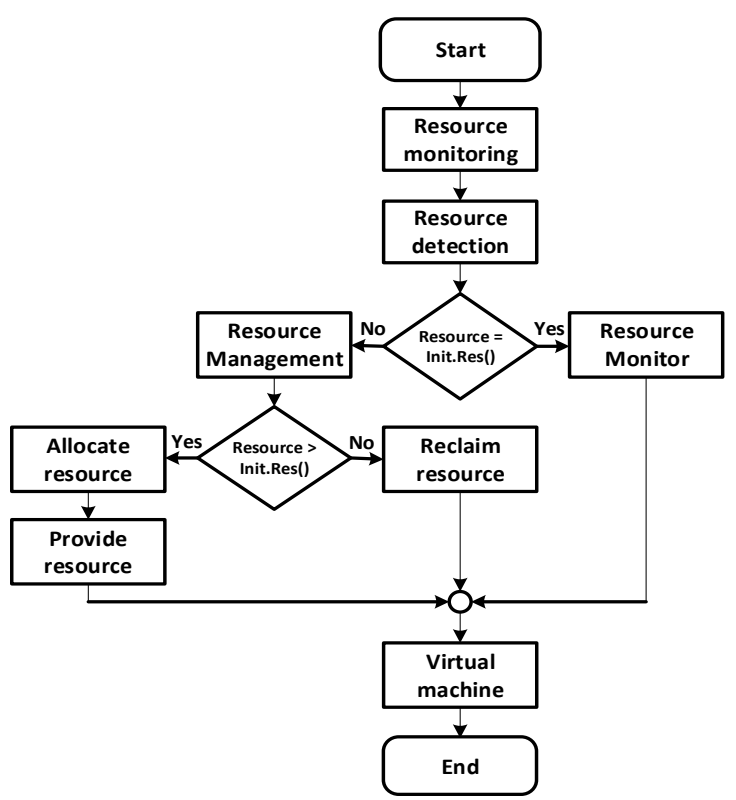

Fig. 7. Process of work.

\section{SCENARIOS}

According to the models of resource allocation, it has shown a term and condition of resource management for Virtual machine in the following circumstances; It assumes that we create virtual machine and configure the default resource (Initial. Res) at 1,000 MB in order to install virtual machine and application enable to provide high quality of service. It has to specify the resource usage not to be more than $80 \%$ of initial.Res. Description of this system operation 
is shown in Fig. 7. Moreover, it possibly defines the resource allocation according to these scenarios.

Scenario 1: Virtual machine uses $800 \mathrm{MB}$ of available resources. According to a term and condition of resource allocation, it starts with resource monitoring of Virtual machine. The detection of resource usage value is $800 \mathrm{MB}$ which is equivalent to the maximum usage value of initial resource from available resource. Thus, it is no need to allocate or restore resource from Virtual machine.

Scenario 2: Virtual machine increases using resource up to $1000 \mathrm{MB}$. According to a term and condition of resource allocation, it starts with resource monitoring of Virtual machine. The detection of resource usage value is $1000 \mathrm{MB}$ which is more than the maximum usage value of initial resource from available resource. (The initial resource value is $800 \mathrm{MB}$ ) That means, the resource allocation has to increase to $200 \mathrm{MB}$ in order to be sufficient for usage. From this scenario, it can sum up that the initial resource should be increased from $1000 \mathrm{MB}$ to $1200 \mathrm{MB}$.

Scenario 3: According to second scenario, Virtual machine decreases using resource down to $600 \mathrm{MB}$. According to a term and condition of resource allocation, it starts with resource monitoring of Virtual machine. The detection of resource usage value is $600 \mathrm{MB}$ which is less than the maximum usage value of initial resource from available resource. (The initial resource value is $1000 \mathrm{MB}$ ) Thus, it is needed to restore resource, $400 \mathrm{MB}$ for proper usage. In conclusion, according to third scenario, the initial resource should be reduced from 1,200 MB to $800 \mathrm{MB}$.

\section{CONCLUSION}

According to the proposed models of resource allocation, it monitors the resource usage of Virtual machine all the time, which effects to the initial resource to be changed according to the actual usage for each time period. This process benefits to resource allocation and restoration to be more appropriate for Virtual machine performance. Moreover, it can support the quality of application services running on Virtual machine by specifying the proper percentage of maximum initial resource as needed.

However, the model of resource allocation indicates that it has to rely on a connection of Hypervisor in order to use physical resource. That means the physical resource should be adequate for a virtual machine running on a Physical hardware.

For guideline for the future research, since this research is to present a concept and models of resource allocation for Virtual machine according to scenarios. Thus, in the future, it is necessary to invent the algorithm for assigning function in each module in order to create resource allocation system.

\section{REFERENCES}

[1] M. Principato, "Virtualization technology and process control system upgrades," in Proc. IEEE-IAS/PCA 52 ${ }^{\text {nd }}$ Cement Industry Technical Conference, 2010, pp. 1-12.
[2] A. Spellmann, K. Erickson, and J. Reynolds, "Server consolidation using performance modeling," IT Professional, pp. 31-36, vol. 5, 2003.

[3] C. Clark, K. Fraser et al., "Live migration of virtual machine," in Proc. $2^{\text {nd }}$ Symposium on Networked Systems Design \& Implementation, 2005, pp. 273-286.

[4] E. Ackaouy. Xen Source ${ }^{\mathrm{tm}}$ The Xen Credit CPU Scheduler. Archive.xenproject. [Online]. Available: www-archive.xenproject.org/files/summit_3/sched.pdf

[5] H. Liu, H. Jin et al., "Hotplug or ballooning: A comparative study on dynamic memory management techniques for virtual machines," IEEE Transactions on Parallel and Distributed Systems, p. 1, 2014.

[6] G. Ning and S. Yongqing, "Research and implementation of resource management system based on xen virtual machine," in Proc. ICCSNT, 2011, vol. 3, pp. 1371-1374.

[7] D. Minarolli and B. Freisleben, "Virtual machine resource allocation in cloud computing via multi-Agent Fuzzy control," in Proc. Third IEEE International Conference, 2013, pp. 188-194.

[8] S. B. Nigmandjanovich and C. Ahn, "Policy-based dynamic resource allocation for virtual machine on xen-enabled virtualization environment," in Proc. ICACC, 2010, vol. 5, pp. 353-355.

[9] D. Minarolli and B. Freisleben, "Distributed resource allocation to virtual machines via Artificial neural networks," in Proc. 22nd Euromicro International Conference, 2014, pp. 490-499.

[10] Y. Song, Y. Sun, and W. Shi, "A Two-Tiers on demand resource allocation mechanism for VM-Based data centers," IEEE, vol. 6, pp. 116-129, 2013.

[11] W. Zhou, S. Yang, J. Fang, X. Niu, and H. Song, "VMCTune: A load balancing scheme for virtual machine cluster using dynamic resource allocation," in Proc. 9th International GCC, 2010, pp. 81-86.

[12] C. Yang, K. Wang, H. Cheng, C. Kuo et al., "Green power management with dynamic resource allocation for cloud virtual machine," in Proc. HPCC, IEEE, 2011, pp. 726-733.

[13] S. Huisman and M. Haverink. Application virtualization comparison chart 2009. [Online]. Available: http://virtualfuture.info/wp-content/uploads/2009/08/ApplicationVirt CompChart2009.pdf

[14] W. Chen, H. Lu et al., "Novel hardware assisted full virtualization technique," in Proc. the 9th International Conference on Young Computer Scientists, pp. 1292-1297, 2008.

[15] K. T. Raghavendra, S. Vaddagiri, N. Dadhania, and J. Fitzhardinge, "Paravirtualization for scalable Kernel-based Virtual Machine (KVM)," in Proc. IEEE International Conference on Cloud Computing in Emerging Markets, 2012, pp. 1-5.

[16] W. Chen, H. Lu et al., "DBTIM: An advanced hardware assisted full virtualization architecture," in Proc. IEEE/IFIP International Conference on Embedded and Ubiquitous Computing, 2008, pp. 399-404.

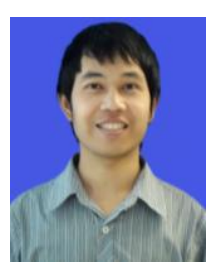

Dolwichit Jannarm graduated with a bachelor degree in computer technology from Rajamangala University of Technology, Phatumtani, Thailand in 2006. He is a master student in information technology and also works as a system engineer in Communication Center at Thai-Nichi Institute of Technology, Thailand.

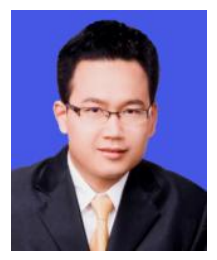

Thongchai Kaewkiriya graduated with a bachelor degree in computer technology and electronic telecommunication engineering from King Mongkut's University of Technology North Bangkok, Thailand in 2000 and Pathumwan Institute of Technology Bangkok, Thailand in 2006. He graduated with a master degree in electrical and information engineering from King Mongkut's University of Technology Thonburi, Thailand in 2005. He graduated with a doctor degree in electrical engineering and information science from Osaka Prefecture University, Japan in 2014. He works as a lecturer in the field of information technology while also looking after the Information and Communication Center at Thai-Nichi Institute of Technology, Thailand. 